\title{
DESIGN OF OVERSAMPLED UNIFORM DFT FILTER BANKS WITH REDUCED INBAND ALIASING AND DELAY CONSTRAINTS
}

N. Grbić, J. M. de Haan, I. Claesson

\author{
Blekinge Institute of Technology \\ Department of Telecommunications \\ and Signal Processing \\ Ronneby, Sweden
}

\author{
S. Nordholm \\ Australian Telecommunications \\ Research Institute \\ Curtin University \\ Perth, Australia
}

\begin{abstract}
Subband adaptive filters have been proposed to avoid the drawbacks of slow convergence and high computational complexity associated with time domain adaptive filters. Subband processing introduces transmission delays caused by the filter bank and signal degradations due to aliasing effects. One efficient way to reduce the aliasing effects is to allow a higher sample rate than critically needed in the subbands and thus reduce subband signal degradation. We suggest a design method, for a uniform DFT filter bank with any over sampling factor, where the total filter bank group delay may be specified, and where the aliasing and magnitude/phase distortions are minimized.
\end{abstract}

\section{INTRODUCTION}

Subband adaptive filtering has arised as an alternative for conventional time domain adaptive filtering, [1]. The main reason is the reduction in computational complexity and the increase in convergence speed for the adaptive algorithm, by dividing the algorithm into subbands, [2]. The computational savings comes from the fact that time domain convolution becomes decoupled in the subbands, at a lower sample rate, [3].

Subband analysis and synthesis is often performed using multirate filter banks, [4]. Non-ideal filters in the filter bank cause aliasing of the subband signals. This aliasing can be cancelled in the synthesis bank when certain conditions are met by the synthesis filters and in the subband processing. However, even if this aliasing is cancelled in this way, the inband aliasing is still present in the subband adaptive filter input signals and, consequently, the adaptive filters are perturbed and the overall performance of the system is reduced, [5].

Several solutions to the subband filtering problem have been suggested in the literature. Non-critical decimation has been suggested in [1], where filter bank delay aspects, and amplitude distortions, have not especially been taken into consideration. The use of cross filters, [5], has been suggested to explicitly filter out the aliasing components. A delayless structure has been proposed in [6], where the actual filtering is performed in the time domain, with consequences of higher computational complexity. The computational complexity also increases significantly with cross band filters. The use of allpass IIR filter banks gives very high sidelobe attenuation and has been shown to be computationally efficient, while keeping the aliasing effects low, [7]. Non-linear phase distortions and appearance of narrowband high energy aliasing terms may be noticed at the subband boundaries with this approach.

We use an uniform DFT modulated FIR filter bank for the subband transformations. Modulated filter banks provide a computationally efficient implementation, due to the polyphase implementation [4], and great design simplicity. The main contribution in this paper is the suggested design method, where the inband and the reconstruction aliasing are minimized simultaneously, while the total filter bank group delay is pre-specified. A numerical comparison of a real room identification shows that both the inband aliasing and the filter bank delay affects the identification accuracy.

\section{THE UNIFORM DFT MODULATED FILTER BANK}

A set of $K$ filters forms a uniform DFT analysis filter bank when they are related to a prototype filter, $h_{0}(n)$, as

$$
\begin{array}{r}
H_{k}(z)=H_{0}\left(z W_{K}^{k}\right)=\sum_{n=-\infty}^{\infty} h_{0}(n)\left(z W_{K}^{k}\right)^{-n} \\
k=0, \cdots, K-1
\end{array}
$$

where $W_{K}=e^{-j 2 \pi / K}$. Each subband signal is decimated by a factor, $D$. An efficient implementation of such filter bank is given in [8]. In order to analyze the magnitude and aliasing effects caused by the filter bank, a direct form realization of the filter bank (see Fig. 1) is beneficial when it comes to uniformity of the derivations for different decimation factors. The subband filters in the synthesis filter bank are the same as for the analysis filter bank, i.e. $F_{k}(z)=H_{k}(z)$. Each branch signal, $V_{k}(z)$, is simply a filtered version of the input signal

$$
V_{k}(z)=H_{k}(z) X(z)=H_{0}\left(z W_{K}^{k}\right) X(z) .
$$

The decimators cause a summation of repeated and expanded spectra of the input signal

$$
X_{k}(z)=\frac{1}{D} \sum_{l=0}^{D-1} V_{k}\left(z^{\frac{1}{D}} W_{D}^{l}\right)
$$




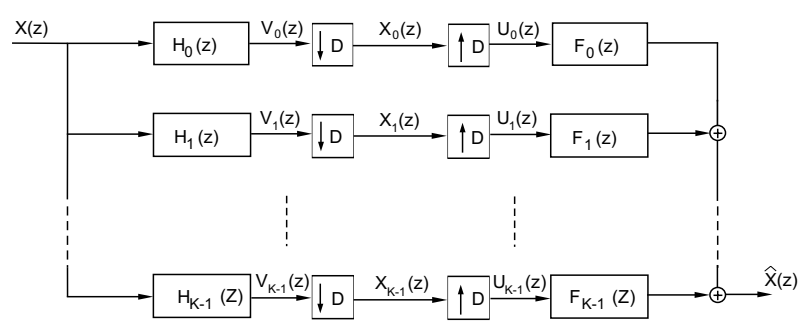

Figure 1: Direct form filter bank realization.

$$
=\frac{1}{D} \sum_{l=0}^{D-1} H_{0}\left(z^{\frac{1}{D}} W_{M}^{k} W_{D}^{l}\right) X\left(z^{\frac{1}{D}} W_{D}^{l}\right)
$$

where $W_{D}=e^{-j 2 \pi / D}$. The interpolators have a compressing effect

$$
U_{k}(z)=X_{k}\left(z^{D}\right)=\frac{1}{D} \sum_{l=0}^{D-1} H_{0}\left(z W_{K}^{k} W_{D}^{l}\right) X\left(z W_{D}^{l}\right) .
$$

By yet another filtering operation by the reconstruction filters we state a relation between the input signal $X(z)$ and the output signal $\hat{X}(z)$

$$
\hat{X}(z)=\sum_{l=0}^{D-1} A_{l}(z) X\left(z W_{D}^{l}\right)
$$

where

$$
A_{l}(z)=\frac{1}{D} \sum_{k=0}^{K-1} H_{0}\left(z W_{K}^{k} W_{D}^{l}\right) H_{0}\left(z W_{K}^{k}\right) .
$$

The transfer functions, $A_{l}(z), \quad l=1, \cdots, D-1$, can be viewed as aliasing gains. The function, $A_{0}(z)$, is the magnitude gain for the original input signal spectrum.

The magnitude and phase response of the overall transfer function, $T(z)$, and the distortion function, $D(z)$, can be described in terms of the repeated spectrum gains, $A_{l}(z)$, as

$$
T(z)=\sum_{l=0}^{D-1} A_{l}(z) \quad D(z)=\sum_{l=1}^{D-1} A_{l}(z) .
$$

For each subband signal, we define a measure of the inband aliasing as the total gain of all repeated spectra in that subband. Due to the modulated structure of the filterbank, the inband aliasing gains will be the same for all subband signals. Thus, we only measure the inband aliasing for the first subband, $D_{0}(z)$, given by

$$
D_{0}(z)=\frac{1}{D} \sum_{l=1}^{D-1}\left|H_{0}\left(z^{\frac{1}{D}} W_{D}^{l}\right)\right| .
$$

The inband aliasing given in Eq. (8) is an upper bound on the actually incurred aliasing term, since cancellation of the terms in the sum may occur. However, filtering in the subbands will alter the cancellation effect and the objective is to minimize the upper bound in order to successfully tackle adaptive filtering in the subbands.

\section{PROTOTYPE FILTER OPTIMIZATION}

We want to find a prototype filter $\mathbf{h}$ which minimizes the overall error function $E(\mathbf{h})$

$$
E(\mathbf{h})=\alpha \epsilon_{1}(\mathbf{h})+\beta \epsilon_{2}(\mathbf{h})+\gamma \epsilon_{3}(\mathbf{h})+\delta \epsilon_{4}(\mathbf{h})
$$

where the factors $\alpha, \beta, \gamma$ and $\delta$ are weight factors and the average amplitude error, $\epsilon_{1}$, is defined as

$$
\epsilon_{1}(\mathbf{h})=\left.\frac{1}{2 \pi} \int_{-\pi}^{\pi}|1-| T\left(e^{j \omega}\right)\right|^{2} \mid d \omega
$$

and the average phase error, $\epsilon_{2}$, is defined as

$$
\epsilon_{2}(\mathbf{h})=\frac{1}{2 \pi} \int_{-\pi}^{\pi}\left|\angle T\left(e^{j \omega}\right)-\angle T\left(e^{j 0}\right)+\tau \omega\right| d \omega
$$

where $\tau$ is the desired group delay of the filter bank response $T(z)$. The average aliasing distortion, $\epsilon_{3}$, is defined as

$$
\epsilon_{3}(\mathbf{h})=\frac{1}{2 \pi} \int_{-\pi}^{\pi}\left|D\left(e^{j \omega}\right)\right|^{2} d \omega
$$

and the average aliasing distortion in the first subband, $\epsilon_{4}$, is defined as

$$
\epsilon_{4}(\mathbf{h})=\frac{1}{2 \pi} \int_{-\pi}^{\pi}\left|D_{0}\left(e^{j \omega}\right)\right|^{2} d \omega .
$$

The minimization of Eq. (9) is a very involved non-linear optimization problem. We suggest an iterative two step procedure, where we optimize Eq. (9) based on two design parameters, a passband boundary frequency, $\omega_{p}$, and a stopband boundary frequency, $\omega_{s}$. For given design parameters, a prototype filter $\mathbf{h}$, can be created using methods outlined in [9] and [10]. This is a complex domain filter design method which allows also for optimization on the group delay. The objective in this design is to minimize the function, $J\left(\omega_{s}, \omega_{p}, \tau\right)$, on a sampled grid of frequencies

$$
J\left(\omega_{s}, \omega_{p}, \tau\right)=\sum_{i=1}^{N}\left|H_{d}\left(\omega_{i}\right)-H\left(\omega_{i}\right)\right|^{2}
$$

where

$$
H_{d}(\omega)= \begin{cases}e^{-j \omega \tau / 2} & \omega \in \Omega_{p} \\ 0 & \omega \in \Omega_{s}\end{cases}
$$

is the desired complex filter specification with the passband region defined as $\Omega_{p}=\left[0, \omega_{p}\right]$ and the stopband region defined as $\Omega_{s}=\left[\omega_{s}, \pi\right]$. The predetermined total desired filter bank group delay is $\tau$, and

$$
H(\omega)=\sum_{n=0}^{L-1} h_{0}(n) e^{-j \omega n}
$$

is the frequency response of the prototype filter.

The objective of the optimization is then,

$$
\left[\omega_{p}, \omega_{s}\right]=\arg \min _{\omega_{p}>0, \omega_{s}>\omega_{p}} E\left(\mathbf{h}\left(\omega_{p}, \omega_{s}, \tau\right)\right) .
$$

The algorithm follows: 
1. Initialization phase. The passband and stopband frequencies are initialized with $\omega_{p, 0}=\frac{\pi}{M}$ and $\omega_{s, 0}=$ $\frac{\pi}{D}$, respectively. Initial step sizes $\zeta_{p, 0}$ and $\zeta_{s, 0}$ are set. Iteration index, $i$, is set to 0 .

2. Design phase. The prototype filter $\mathbf{h}\left(\omega_{p, i}, \omega_{s, i}, \tau\right)$, is designed such that Eq. (14) is minimized.

3. Optimization phase. The filter specification frequencies $\omega_{p}$ and $\omega_{s}$ are adapted according to

$$
\begin{aligned}
\omega_{p, i+1} & =\omega_{p, i}-\zeta_{p, i} \operatorname{sgn}\left(\dot{\omega}_{p, i}\right) \\
\omega_{s, i+1} & =\omega_{s, i}-\zeta_{s, i} \operatorname{sgn}\left(\dot{\omega}_{s, i}\right)
\end{aligned}
$$

where

$$
\dot{\omega}_{p}=\frac{E_{i}-E_{i-1}}{\omega_{p, i}-\omega_{p, i-1}} \quad \dot{\omega}_{s}=\frac{E_{i}-E_{i-1}}{\omega_{s, i}-\omega_{s, i-1}}
$$

where we have used discretized approximations of the gradient. The step sizes are exponentially decreased. Index $i$ is increased by one and steps $\mathbf{2}$ and $\mathbf{3}$ are continued until a stop criterion is met.

\section{EVALUATION}

We have designed two critical and two non-critical decimated filter banks with 32 subbands and each with decimation factor 32 and 16, respectively. The length of the prototype filter is 128 and the group delay is specified as $\tau=128$ and $\tau=64$. Table 1 shows the final distortion measures after optimization, for the four scenarios. We evaluate the performance of the subband implementations in the case of a real room impulse response estimation. A white noise sequence is emitted through a loudspeaker in a conference room and by using a microphone observation as a desired signal, we identify the acoustic path, see Fig. 2. We use the least squares estimation method, [2], individually in each subband, and compare the fullband FIR filter identification with subband identifications achieved with the filter banks given in Table 1. The average spectral error of the estimations, are given in Table 2, together with A FFT filter bank implementation. The system responses of the critically decimated filter bank identifications are shown in Fig. 3, while Fig. 4 show the response of the non-critically decimated filter banks, together with the real room system response. It can be seen that the variations are much larger with the critically decimated subband implementation, especially at the subband boundaries.

The estimation accuracy for the non-critical decimated filter banks are close to the fullband solution and significantly better than the critically decimated cases.

\section{CONCLUSIONS}

We have proposed an efficient design method for an uniform DFT filter bank with the possibility of a prespecified filter bank group delay. The optimization minimizes the inband aliasing components as well as the overall filter bank transfer function's phase and amplitude deviation. A real room transfer function estimation shows that the accuracy is dependent on both the group delay and the aliasing effects. Subband oversampling decreases the inband aliasing, which in turn increases the estimation accuracy. The gain with

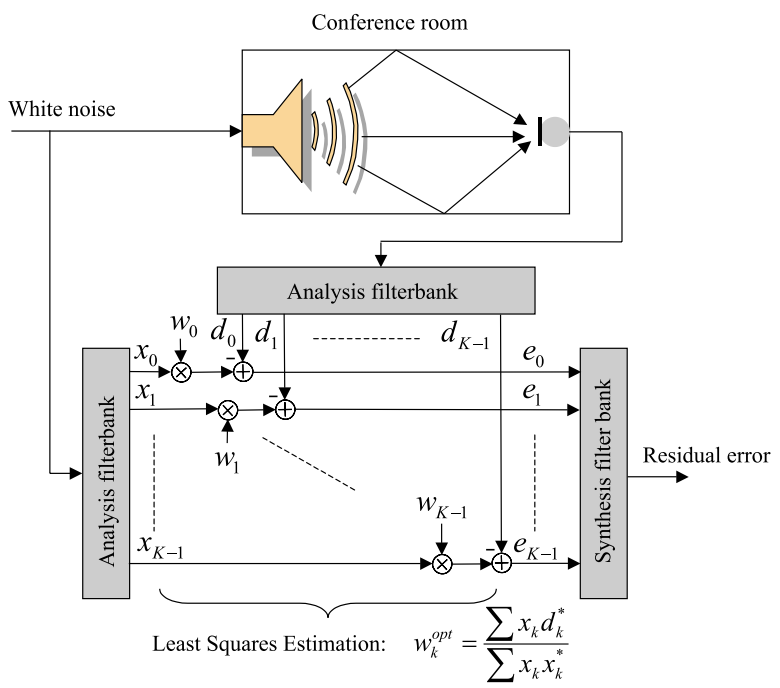

Figure 2: Subband room impulse response identification.

over sampling is more significant when reduced delay filter banks are used.

\begin{tabular}{ll||rr|rr}
\hline \hline \multicolumn{2}{c}{$L=128, M=32$} & \multicolumn{2}{c}{$\tau=128$} & \multicolumn{2}{c}{$\tau=64$} \\
\hline \hline$D=32$ & $\epsilon_{1}$ & -12.56 & $\mathrm{~dB}$ & -3.42 & $\mathrm{~dB}$ \\
& $\epsilon_{2}$ & 0.03 & $\mathrm{rad}$ & 0.76 & $\mathrm{rad}$ \\
& $\epsilon_{3}$ & -44.57 & $\mathrm{~dB}$ & -43.52 & $\mathrm{~dB}$ \\
& $\epsilon_{4}$ & -12.28 & $\mathrm{~dB}$ & -9.48 & $\mathrm{~dB}$ \\
\hline \multirow{4}{*}{$D=16$} & $\epsilon_{1}$ & -21.55 & $\mathrm{~dB}$ & -9.85 & $\mathrm{~dB}$ \\
& $\epsilon_{2}$ & 0.02 & $\mathrm{rad}$ & 0.05 & $\mathrm{rad}$ \\
& $\epsilon_{3}$ & -79.22 & $\mathrm{~dB}$ & -68.02 & $\mathrm{~dB}$ \\
& $\epsilon_{4}$ & -48.31 & $\mathrm{~dB}$ & -36.58 & $\mathrm{~dB}$ \\
\hline \hline
\end{tabular}

Table 1: Average distortion measures, $\epsilon_{1}, \ldots, \epsilon_{4}$ for critical and non-critical decimation with two specified delay cases.

\begin{tabular}{l||c|c|}
\hline \hline$L=128, M=32$ & $\tau=128$ & \multicolumn{2}{|c}{$\tau=64$} \\
\hline \hline$D=32$ & $-8.17 \mathrm{~dB}$ & $-7.74 \quad \mathrm{~dB}$ \\
\hline$D=16$ & $-8.81 \mathrm{~dB}$ & $-9.16 \mathrm{~dB}$ \\
\hline \hline FFT Filter Bank & \multicolumn{3}{|c}{$-5.20 \mathrm{~dB}$} \\
\hline \hline Fullband & \multicolumn{3}{|c}{$-9.84 \mathrm{~dB}$} \\
\hline \hline
\end{tabular}

Table 2: Average spectral error of least squares solutions to a system identification of an acoustic path in a conference room. Four subband cases with 32 weights and an FFT filter bank are compared to the fullband solution.

\section{REFERENCES}

[1] W. Kellermann, "Analysis and design of multirate systems for cancellation of acoustic echoes," in Proc. ICASSP'88, pp. 2570-2573.

[2] S. Haykin, "Adaptive Filter Theory," Prentice Hall Int. Inc., 1996, ISBN 0-13-397985-7. 


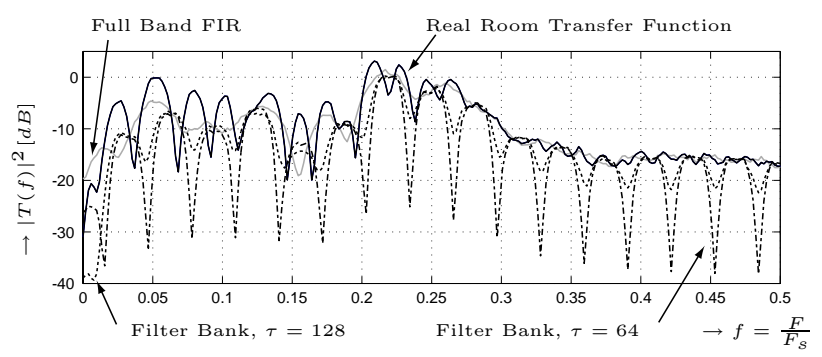

Figure 3: System responses of the real room transfer function estimates for the fullband implementation and the critically decimated subband implementations, $L=128, M=$ $32, D=32$. It can be seen that the identification error is relatively large.

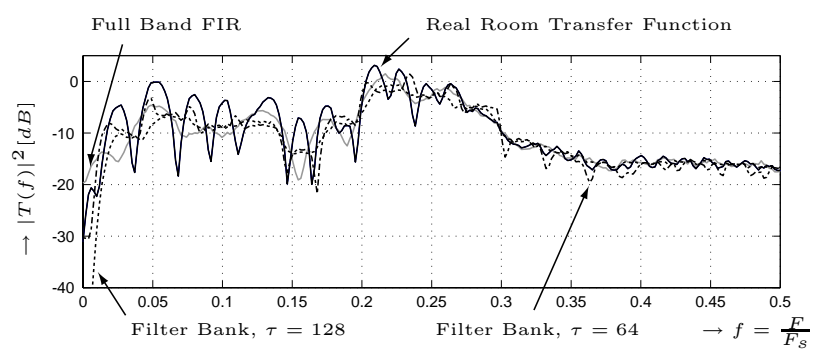

Figure 4: System responses of the real room transfer function estimates for the fullband and the non-critically decimated subband implementations, $L=128, M=32, D=$ 16. It can be seen that the identification error is reduced compared to Fig. 3.

[3] P. P. Vaidyanathan, "Orthonormal and biorthonormal filter banks as convolvers, and convolutional coding gain," IEEE Trans. on Signal Processing, vol. 41, no. 6, pp. 2110-2130, Jun. 1993.

[4] P. P. Vaidyanathan, "Multirate Systems and filter banks," Prentice Hall, 1993.

[5] A. Gilloire, M. Vetterli, "Adaptive filtering in subbands with critical sampling: analysis, experiments, and application to acoustic echo cancellation," IEEE Transactions on Signal Processing, pp. 1862-1875, vol. 40, issue 8, Aug. 1992.

[6] D. R Morgan, J. C. Thi, "A delayless subband adaptive filter architecture," IEEE Trans. Signal Processing, vol. 43, pp. 1819-1830, 1995.

[7] P. A. Naylor, O. Tanrikulu A. G. Constantinidis, "Subband Adaptive Filtering for Acoustic Echo Control using Allpass Polyphase IIR filter banks," IEEE Trans. on Speech and Audio Processing, vol. 6, no. 2, Mar. 1998.

[8] N. Grbić, "Speech Signal Extraction - A Multichannel Approach," Blekinge Institute of Technology, pp. 7275, Nov. 1999, ISBN 91-630-8841-X.
[9] T. W. Parks, C. S. Burrus, "Digital Filter Design," John Wiley and Sons, Inc., 1987, ISBN 0-471-82896-3.

[10] D. R. Xiangkun Chen, T. W. Parks, "Design of FIR filters in the Complex Domain," IEEE Trans. on Acoustics, Speech and Signal Processing, vol. ASSP-35, no. 2, Feb. 1979. 